\title{
Entrelacs
}

Cinéma et audiovisuel

$11 \mid 2014$

La Voix

\section{Les voix des héroïnes de Geraldine Chaplin dans le cinéma de Carlos Saura ou la mise en lumière d'une collaboration artistique}

The voices of Geraldine Chaplin's heroines in the cinema of Carlos Saura:

shedding light on an artistic collaboration

\section{Arnaud Duprat de Montero}

\section{(2) OpenEdition}

\section{Journals}

Édition électronique

URL : http://journals.openedition.org/entrelacs/912

DOI : 10.4000/entrelacs.912

ISSN : 2261-5482

Éditeur

Éditions Téraèdre

\section{Référence électronique}

Arnaud Duprat de Montero, «Les voix des héroïnes de Geraldine Chaplin dans le cinéma de Carlos

Saura ou la mise en lumière d'une collaboration artistique », Entrelacs [En ligne], 11 | 2014, mis en ligne le 31 décembre 2014, consulté le 30 avril 2019. URL : http://journals.openedition.org/entrelacs/912 ;

DOI : 10.4000/entrelacs.912

Ce document a été généré automatiquement le 30 avril 2019

Tous droits réservés 


\title{
Les voix des héroïnes de Geraldine Chaplin dans le cinéma de Carlos Saura ou la mise en lumière d'une collaboration artistique
}

\author{
The voices of Geraldine Chaplin's heroines in the cinema of Carlos Saura: \\ shedding light on an artistic collaboration
}

Arnaud Duprat de Montero

La collaboration artistique entre le cinéaste espagnol Carlos Saura et l'actrice angloaméricaine Geraldine Chaplin est susceptible d'attirer notre attention à plus d'un titre. Tout d'abord, elle nous interpelle en raison de sa durée et de sa fréquence : s'étendant de 1967 à 1979, cette collaboration concerne huit films ${ }^{1}$, c'est-à-dire la quasi-totalité de la production du réalisateur sur cette période, hormis el Jardín de las delicias (le Jardin des délices, 1970), film dédié à l'actrice où elle fait une courte apparition muette, et la Prima Angélica (la Cousine Angélique, 1974) qui est, cette fois-ci, dédié à ses parents, Oona O'Neill et Charles Chaplin. De plus, la variété des héroïnes est remarquable : Geraldine Chaplin a incarné pour Carlos Saura des femmes-objet libertaires comme des épouses à l'apparence soumise, aussi bien anglaises qu'espagnoles. Enfin, nous devons souligner la richesse vocale de ces créations. Doublées par plusieurs actrices, conservant la voix de Geraldine Chaplin enregistrée en son direct, ou même postsynchronisées par cette dernière dans une langue différente, les héroïnes affichent là encore une variété qu'il serait réducteur de considérer comme un choix palliant l'apprentissage progressif de la langue espagnole par l'actrice. Ce phénomène semble plutôt interroger la place occupée par Geraldine Chaplin dans l'œuvre alors essentiellement espagnole de Carlos Saura ${ }^{2}$. Effectivement, en étant actrice pour David Lean, Robert Altman, James Ivory, Jacques Rivette, Alain Resnais ou bien encore Michel Deville ${ }^{3}$, Geraldine Chaplin a toujours été une personnalité artistique à mi-chemin entre plusieurs cultures et ces voix pourraient donc être symptomatiques de l'impossibilité d'intégrer pleinement sa créativité à cette époque de l'œuvre saurienne. Cependant, nous ne pouvons nier que Geraldine Chaplin en est 
aujourd'hui encore l'icône féminine. Afin de tenter de mieux comprendre ce paradoxe, il conviendra, dans un premier temps, d'interroger cette variété vocale et voir dans quelle mesure la créativité de l'actrice s'est adaptée à son caractère a priori artificiel. De plus, alors qu'elle n'est pas doublée dans ses films français ou anglophones, nous évaluerons comment la coprésence, dans ce système, de sa voix véritable avec son léger accent britannique, a pu nourrir l'affirmation de sa persona ${ }^{4}$ et permettre, toujours de manière paradoxale, une certaine hispanisation de celle-ci. Enfin, nous nous pencherons plus particulièrement sur l'évolution de la mise en scène vocale des héroïnes - de simples voix in à des voix over narratrices - qui semble encore mettre en lumière autant de frictions créatrices qu'une simple intégration de Geraldine Chaplin à la poétique saurienne.

$2 \mathrm{Si}$, en octobre 1967, les critiques sont nombreux à remarquer la rupture que représente Peppermint Frappé dans la filmographie de Carlos Saura ${ }^{5}$, l'importance nouvelle du féminin grâce à la figure duelle Elena/Ana en constitue une des principales explications. En acceptant d'interpréter ces deux personnages si opposés, Geraldine Chaplin incarne une conception dichotomique du féminin présentée ainsi par Carlos Saura :

3 «Peppermint Frappé est l'histoire d'un homme qui hésite entre ces deux femmes. D'un côté, il est fasciné par la femme-objet, une fascination que je partage et que nous partageons tous en raison de notre éducation... Il suffit de regarder n'importe quel magazine de mode - Vogue, Marie-Claire - et les publicités à la télévision. Cette femmeobjet, qui doit être belle, coiffée et apprêtée, avec des talons aiguilles, etc., est comme un jouet. De l'autre côté, vous avez la femme qui, tout simplement, doit travailler pour vivre et qui est beaucoup plus proche d'une réalité concrète. Et ceci est Peppermint Frappé »6.

4 En voulant transformer l'espagnole Ana, une modeste assistante médicale à l'apparence humble et innocente, en un sosie de la britannique Elena, jeune bourgeoise oisive et sophistiquée, Julián - José Luis López Vázquez - manifesterait donc une obsession masculine générationnelle pouvant être lue comme la métaphore d'un cinéaste pygmalion qui veut créer de toutes pièces son actrice idéale, à partir d'une jeune interprète en devenir ${ }^{7}$. Cette idée de la recréation artificielle s'appuie en grande partie sur la décision de doubler Geraldine Chaplin dans le rôle d'Ana ${ }^{8}$. Comme l'a résumé Jacqueline Nacache, « dompter les voix reste la grande affaire du cinéma [...] Entre la voix et le corps divorcés de l'acteur, se glisse la main de fer du cinéaste $»^{9}$. Alors qu'Elena, qui ne se soumet pas aux fantasmes de Julián, conserve la vraie voix de l'actrice, Ana, à la docilité finalement inquiétante, se voit attribuer celle d'une autre comédienne. Certes, dans la mesure où le public espagnol était à l'époque habitué aux doublages des films étrangers, ce dernier choix pouvait parfaitement être accepté. Cependant, la coprésence de la véritable voix de Geraldine Chaplin en Elena, rend l'artifice incontournable, d'autant plus que, suite au grand succès de Doctor Zhivago (Le docteur Jivago, David Lean, 1965), le public identifiait à priori Geraldine Chaplin comme une actrice étrangère et anglophone. Enfin, ayant reconnu dans ce dernier film la chevelure brune comme lui étant naturelle, les spectateurs peuvent percevoir la blondeur d'Elena comme un leurre et cette hérö̈ne comme une représentante caricaturale du Swinging London. Si nous adhérons à la théorie de Yannick Lemarié qui considère que tout personnage cinématographique constitue une figure acteur/acté - « comme les deux faces inséparables d'une réalité unique, placée sous le regard indispensable du spectateur » car ce dernier « part du personnage pour revenir à un moment ou à un autre au comédien. Impossible pour lui d'ignorer le visage sous le masque. Impossible d'ignorer le masque sur le visage $»^{10}-$, nous pouvons dire que la figure proposée par Elena et Ana est frappée d'artificiel car l'«acté » y serait contrarié 
par «l'actrice » qui domine toujours. De plus, dans la mesure où le doublage intervient dans le cas de l'héroïne censée incarner une certaine authenticité hispanique - tout comme une authenticité par rapport à la persona de l'actrice alors fondée sur son rôle de jeune femme pure et effacée de Doctor Zhivago -, le procédé peut nous évoquer les propos d'Edgar Morin qui voit dans le doublage la démonstration de "l'inutilité limite de l'acteur $»^{11}$ car le personnage peut être recréé par le cinéaste au montage son, et présente ainsi Geraldine Chaplin davantage comme une créature que comme une créatrice. D'ailleurs, dans le film suivant, Stress es tres tres, l'actrice est encore blonde - même si cette fois-ci elle retire sa perruque à la fin du film - et doublée - par une autre actrice que celle de Peppermint Frappé, empêchant ainsi toute cohérence vocale - dans un personnage de bourgeoise espagnole, oisive et superficielle, une femme-objet qui trouvera par la suite un écho chez les héroïnes de la Madriguera jusqu’à los Ojos vendados, en passant, dans une moindre mesure, par la mère de Cría cuervos...

5 Pour autant, Teresa - l'héroïne de Stress es tres tres - marque une rupture car elle refuse de n'être qu'une femme-objet («Je ne suis pas un objet», dit-elle face à son miroir) et laisse deviner, derrière ses lunettes de soleil et sa blondeur artificielle, une blessure (aime-t-elle son mari ? Le trompe-t-elle ?) qui permet d'abandonner progressivement la dimension caricaturale d'Elena et de développer l'énigme inquiétante d'Ana. Cet enrichissement psychologique se confirme à l'occasion de la Madriguera. Carlos Saura souligna l'implication et l'apport créatif de Geraldine Chaplin dès l'écriture de ce nouveau scénario, dans la continuité du précédent film ${ }^{12}$. Jeune épouse bourgeoise qui vient de perdre ses parents dans un accident d'avion, Teresa oscille entre la femme-objet, la femme-enfant, le fantasme sexuel... pour dépasser toujours ces clichés et incarner une multiplicité riche, insaisissable et inquiétante qui débouchera sur le meurtre de Pedro, son mari - Per Oscarsson - puis sur son suicide. Ce final tragique peut être mis en doute en raison d'un subtil et complexe système énonciatif qui mêle progressivement le rêve et la réalité des personnages, de la même façon que plusieurs informations interdites aux spectateurs ou communiquées tardivement, nous incitent à reconsidérer à plusieurs reprises les événements et les protagonistes - le meilleur exemple en est ce bouquet de roses qui nous fait croire à l'infidélité de la jeune femme, avant d'apprendre qu'il a été envoyé par Pedro sous un faux nom. L'implication de Geraldine Chaplin dans le processus créatif ne renie donc pas la conception féminine duelle de Carlos Saura, mais lui apporte une profondeur et une maturité qui impose une nouvelle thématique - la déréliction du couple, dans la continuité de l'union malsaine de Julián et Ana à la fin de Peppermint Frappé - et, en raison du caractère insaisissable de l'hérö̈ne, accompagne l'apparition d'une structure de récit où les repères spatio-temporels et énonciatifs se troublent. De plus, si Elena et Teresa - Stress es tres tres - faisaient déjà preuve d'un certain infantilisme et d'un goût marqué du jeu, l'hérö̈ne de la Madriguera, en s'amusant à faire revivre la petite fille qu'elle a été, ou en endossant la personnalité de plusieurs proches, développe des situations qui créent des effets de miroir avec une démarche "actorale ", et mettent en lumière les qualités dramatiques de Geraldine Chaplin. Teresa incarne plusieurs images féminines tout comme l'actrice peut donner vie à plusieurs rôles et renaître à chaque fois, même après la mort. Ceci est le cas à la fin de la Madriguera avec le premier suicide feint de Teresa, tout comme cela sera également le cas d'Ana qui reviendra dans Mamá cumple cien años, malgré son meurtre, apparemment fantasmé, à la fin d'Ana y los lobos. Ce phénomène trouvera son accomplissement dans los Ojos vendados où Emilia devient comédienne et dont la mort finale sur scène peut être mise en doute (les terroristes qui tirent sur elle sont-ils véritables, fantasmés ou font-ils partie du 
spectacle ?). La Madriguera serait donc le film où la créativité de Geraldine Chaplin s'impose dans l'œuvre de Carlos Saura en assumant une importance auctoriale et il n'est pas anodin qu'elle joue ici avec sa voix véritable. Étant née de parents espagnols mais ayant des origines anglaises par sa mère, Teresa s'exprime dans un espagnol grammaticalement correct avec un léger accent britannique.

6 Si l'apport créatif de Geraldine Chaplin nourrit et enrichit l'œuvre de Carlos Saura, il a également comme conséquence d'affirmer la persona de l'actrice. Ce phénomène s'accompagne de la présence nécessaire de la voix. Martin Shingler écrit que :

«[...] la voix est un trait distinctif définissant la persona de la star [...] ce n'est peutêtre pas surprenant que Tytti Soila, dans l'introduction du livre Stellar Encounters, precise qu'un grand nombre des participants à son recueil d'articles sur les stars européennes, identifie la voix (avec les yeux) comme "l'expression la plus remarquable d'une performance d'acteur" $»^{13}$.

7 De plus, comme l'a souligné Jean-Pierre Ryngaert, la voix est nécessaire à la reconnaissance des capacités "actorales" car, "plus que simple véhicule du langage, la voix révèle le corps. En témoignent la crainte et le refus si souvent invoqués d'entendre celle de Greta Garbo afin de garder intact le mythe de la déesse détachée des contingences $»^{14}$. En révélant le corps, la voix libère ce dernier et cette liberté appelle une nouvelle écriture filmique. Dans Peppermint Frappé, Elena ne cesse de courir, d'arpenter les pièces où elle se trouve et même de danser. Elle se différencie ainsi d'Ana qui, face à Julián, est filmée assise ou allongée et le seul véritable mouvement qu'elle effectue est quand le héros l'oblige à s'installer sur son rameur d'appartement. D'une héroïne à l'autre, nous passons d'une caméra presque fixe à une caméra virevoltante ${ }^{15}$. De la même façon, la britannique Ana, dans Ana y los lobos, jouit également de cette liberté de mouvement et arpente seule la nature qui entoure la maison familiale d'où ne sortent les autres femmes qu'accompagnées par les hommes. Cette liberté du corps relève donc de la liberté créatrice de l'acteur, comme nous le comprenons lors des cours d'art dramatique auxquels assiste l'apprentie comédienne Emilia et où elle doit apprendre à défaire son corps de toute sa rigidité. Soulignons que cet exercice n'est pas muet. La voix émancipe le corps des limites du cadre cinématographique. Ainsi, la mère de Cría cuervos ne cesse d'entrer et de sortir du champ de la caméra, alors que sa fille adulte - interprétée par une Geraldine Chaplin doublée - est toujours assise et filmée par une caméra fixe qui la cadre dans un plan rapproché quelque peu claustrophobe ${ }^{16}$. La voix, qui accomplit la persona en permettant l'expression de toute la richesse du jeu - car vocal et corporel -, est accompagnée, dans le cas de la mère, de l'accent britannique de l'actrice. Ce dernier accentue le phénomène observé précédemment, comme le souligne Jean-Pierre Ryngaert : « La voix ne va pas non plus sans accent, sauf à la raboter, à l'uniformiser et lui dénier ainsi son origine corporelle et son inscription socioculturelle $»^{17}$. Si le doublage doit donc être évité afin d'affirmer la créativité de l'actrice qui va déterminer l'écriture filmique, la maitrise de la langue espagnole est nécessaire pour éviter de jouer des rôles d'étrangères dans un univers si espagnol. L'accent empêcherait-il ce processus? La persona de Geraldine Chaplin, construite justement sur ce caractère international rappelons que, dans la Madriguera, et dans la continuité d'une carrière entre la France et les Etats-Unis, l'actrice s'y exprime également en anglais et en français -, se verrait-elle empêchée d'intégrer pleinement l'univers saurien?

8 Dans son ouvrage Star Studies, A Practical Guide, Martin Shingler rappelle que la persona d'un acteur n'est jamais fixe et évolue constamment par rapport aux idéologies et au phénomène de l'identité nationale ${ }^{18}$. Malgré le caractère international de sa persona, 
Geraldine Chaplin peut donc trouver sa place dans l'œuvre de Carlos Saura, à condition d'entrer en correspondance avec sa poétique. Les mots de Michel Chion apportent un éclairage pertinent au doublage de Peppermint Frappé et Stress es tres tres :

«Le son rôde autour de l'image comme la voix autour du corps. Ce qui l'empêche de s'y fixer, ce sont les mots perdus ou supprimés, ceux de la profération originelle dont témoigne l'image. Ces mots ne peuvent être oubliés. Le doublage fabrique un palimpseste, sous lequel court un texte fantôme $»^{19}$.

La rencontre de la bande-son et de la bande-image permet de mêler deux temporalités, le mouvement des lèvres de l'actrice à l'écran renvoyant à un passé actualisé grâce aux nouvelles voix. Ce phénomène trouve pleinement sa place dans la poétique saurienne où le présent rencontre si fréquemment le passé dès el Jardín de las delicias où Antonio - José Luis López Vázquez - revisite les souvenirs de son enfance en compagnie du fantôme de sa tante - Lina Canalejas. Bien plus qu'un simple recours technique rendu obligatoire par une actrice étrangère, le doublage dans ces films peut apparaître comme symptomatique de ce phénomène en gestation, d'autant plus que les deux protagonistes masculins de Stress es tres tres sont également postsynchronisés - avec les véritables voix de leurs acteurs - et que La madriguera, film tourné en anglais, fut entièrement doublé ensuite par Geraldine Chaplin. Même si c'est sa voix que nous entendons, la postsynchronisation parfois approximative produit les mêmes impressions. Par la suite, Carlos Saura opte pour le son direct, mais l'accent de l'actrice peut prolonger ces effets ${ }^{20}$. Il est significatif qu'Alain Fleischer définisse l'accent comme la «trace d'une langue fantôme [reprenant ainsi le mot de Chion] absentée, inactuelle, cachée dans la profondeur de champ phonétique et acoustique d'une langue présente, actualisée dans la parole $»^{21}$. Ce phénomène pourrait bien expliquer le fait que, après Ana y los lobos où Geraldine Chaplin incarne à nouveau une Britannique, la mère de Cría cuervos et Elisa dans Elisa, vida mía soient espagnoles. Dans les deux films, aucun élément ne laisse supposer des origines étrangères, d'autant plus que les familles des héroïnes apparaissent et les sœurs sont interprétées par des actrices espagnoles qui parlent sans le moindre accent. Le clou est enfoncé dans la mesure où le doublage de la fille de Cría cuervos aurait pu nous rappeler que cet accent espagnol est impossible à l'actrice et où, dans Elisa, vida mía, l'héroïne, aidant son père - Fernando Rey - à comprendre un poème anglais, en lit quelques vers dans une prononciation parfaite, sans que cela constitue une incohérence gênante.

Cría cuervos et Elisa, vida mía représenteraient en ce sens un certain accomplissement dans la collaboration entre Carlos Saura et Geraldine Chaplin. Cependant, dans los Ojos vendados , Emilia présente à nouveau un nom de famille - Spencer - qui fait d'elle une étrangère, avant de revenir à un autre personnage d'Anglaise dans Mamá cumple cien años ${ }^{22}$. Cría cuervos et Elisa, vida mía ne seraient donc pas le terrain artistique où le cinéaste et son actrice entreraient harmonieusement en correspondance. En effet, les deux films voient leurs récits marqués par de multiples frictions mises en lumière par les voix. Le fait que, dans Cría cuervos et Elisa, vida mía, la voix des héroïnes de Geraldine Chaplin ait accès pour la première fois à l'espace off ${ }^{23}$ et assume des responsabilités narratrices pouvait pourtant appuyer cette idée d'accomplissement dans la collaboration. Dans le premier film, la voix over $^{24}$ de la fille intervient à trois reprises sur des images du passé. Si, la première fois, l'héroïne disparait de l'écran pour laisser la place à de vieilles photographies de sa mère sur lesquelles elle continue de nous faire partager ses souvenirs, la deuxième fois, sa voix introduit un récit secondaire constitué de la scène où, enfant, elle voit sa mère se tordant de douleur sur son lit. Enfin, la troisième fois, sa voix over devient pleinement narratrice d'un récit secondaire représentant à l'écran une visite chez des amis de sa tante. De la 
voix in du plan fixe d'Ana adulte évoqué précédemment, nous passons à une voix narratrice au présent, donnant l'impression de pouvoir assumer tout le récit du film, alors que ses premières apparitions semblaient présenter l'héroïne comme un flashforward inséré dans le récit du narrateur premier. Cette évolution prend alors les allures d'un rapport de force avec cette dernière instance narratrice pouvant être anthropomorphisée sous les traits du cinéaste ${ }^{25}$. Remarquons tout de même que cette opposition entre réalisateur et actrice est encore faussée dans la mesure où, dans Cría cuervos, la voix féminine qui assume ces responsabilités n'est pas celle de Geraldine Chaplin, mais la voix de l'actrice de doublage, cette voix «vissée » - pour reprendre le mot de Marguerite Duras ${ }^{26}$ - artificiellement sur l'image de l'héroïne par le cinéaste. En ce sens, Elisa, vida mía marque une évolution dans le processus puisque la voix de Geraldine Chaplin y devient over et narratrice de récits secondaires. Elle y assume toutes les fonctions possibles de la voix au cinéma : in, over, lectrice (quand on sait qu'elle lit un texte) et narratrice (quand sa voix over semble prendre en charge le récit). Cependant, elle entre à nouveau en opposition avec une autre voix assumant les mêmes caractéristiques, celle de son père. Cette voix masculine et les oppositions énonciatrices qui naissent de la mise en scène avec celle de sa fille, pourraient facilement métaphoriser les démarches artistiques et auctoriales du cinéaste et de son actrice ${ }^{27}$. Si les voix s'opposent, dans une structure de récit infiniment complexe, en introduisant des récits secondaires et en semblant assumer l'identité de l'autre, cette mise en scène vocale laisse également supposer une certaine correspondance identitaire entre les deux personnages, dans la mesure où les voix lisent des textes écrits à une première personne difficilement reconnaissable, où le père et la fille s'exprimeraient eux-mêmes ou s'identifieraient à l'autre, pour, au final, assumer éventuellement la narration du récit premier dans son ensemble. Au sein de cette métaphore renvoyant, nous l'avons dit, au cinéaste et à son actrice, la fusion identitaire va de pair avec des frictions répétées entre deux démarches artistiques bien différenciées du fait que nous avons ici une voix féminine face à une voix masculine. En effet, Jean Châteauvert observe que, au cinéma :

« Traditionnellement, la femme a été cantonnée dans certains rôles, dans certaines fonctions à l'intérieur de l'économie narrative. Ce sexisme est particulièrement fort pour ce qui est de la narration et du commentaire over, traditionnellement réservé aux hommes. Pascal Bonitzer lie cette absence de la voix féminine à l'idéologie dominante tandis que Mary Ann Doane explique cette exclusion par des motifs psychanalytiques, la voix d'autorité étant celle du père, celle de la mère étant reléguée à une fonction réconfortante sans véritable autorité ${ }^{28}$.

11 Alors que la voix de Fernando Rey semble légitimement pouvoir incarner une instance narratrice, celle de Geraldine Chaplin dans ce même rôle, paraît plus subversive et relever d'une certaine usurpation.

Néanmoins, précisons bien que, si cette mise en scène des voix dans Elisa, vida mía met en lumière une structure de récit complexe, cette dernière était déjà à l'œuvre dans les films précédents, depuis la Madriguera, premier film où l'actrice a dépassé son simple statut d'interprète. Les deux protagonistes y étaient les énonciateurs secondaires de scènes oniriques qui s'inséraient dans le récit premier sans crier gare, leur véritable nature étant explicitée seulement avec le réveil des personnages. Leurs fantasmes, également narrés en voix in, permettaient déjà de s'initier aux mélanges spatio-temporels et entre réel et imaginaire. De la même façon, à la fin de Ana y los lobos, Ana et Fernando - Fernando Fernán Gómez -, en raison de leur réveil difficile dans la grotte, pourraient être éventuellement les énonciateurs de tout ce final cauchemardesque qui, malgré certaines 
similitudes avec un rêve antérieur du personnage masculin, peut être compris comme réel, jusqu'à la réapparition d'Ana au début de Mamá cumple cien años. De manière plus manifeste, l'affirmation progressive d'Ana dans Cría cuervos comme narratrice du récit entier trouble, nous l'avons dit, les repères spatio-temporels. Le récit de l'enfance, dans la mesure où les personnages sont habillés à la mode de 1976, appartiendrait au présent et les apparitions de la fille adulte au futur. Cependant, dans la mesure où son imagination se mêle à son récit (les apparitions de la mère sont-elles un fantasme d'Ana enfant énoncés par cette dernière au sein du récit du narrateur premier, d'Ana adulte qui se remémore un fantasme enfantin modifié sur le moment par son imagination, ou un fantasme inventé de toutes pièces?), cette mode actuelle pourrait en être le fruit (d'autant plus que, quelque soient les scènes, la mère y porte toujours la même robe), une actualisation de ses souvenirs par rapport au présent, et son enfance renvoyer à une époque antérieure à 1976. Le film ne nous apportera pas la réponse, mêlant ainsi à jamais les époques, les lieux et les sources énonciatrices. Ces frictions créatrices mises en lumière par les voix des héroïnes de Geraldine Chaplin, si elles ne semblent pas relever d'une collaboration artistique dénuée d'oppositions entre le cinéaste et son actrice, ont donc accompagné l'écriture filmique des œuvres vers sa maturité et son accomplissement 29 .

Geraldine Chaplin assumerait, dans l'œuvre cinématographique de Carlos Saura, un rôle auctorial essentiel qui touche même les films où elle n'apparaît pas ou peu, comme el Jardín de las delicias ou la Prima Angélica. Bien plus que la simple tentative d'une actrice anglo-américaine qui se fait une place dans la filmographie d'un réalisateur espagnol et dans une culture qui n'est pas la sienne, les voix multiples et variées - doublées, postsynchronisées, directes - qui sont attribuées à ses héroïnes, nous racontent l'évolution d'une collaboration artistique entre cinéaste et actrice et surtout la révélation de la poétique de ce cinéaste par l'arrivée de cette actrice dans sa filmographie. Si ces voix multiples peuvent être perçues comme le symptôme d'oppositions créatrices entre deux artistes - des oppositions bien réelles à en croire les confidences de Carlos Saura -, elles nous révèlent tout de même que Geraldine Chaplin n'a pas atteint ce statut auctorial dans l'œuvre en s'opposant simplement, mais en faisant vraisemblablement preuve d'une compréhension fine de la démarche créatrice du réalisateur, qui a supposé une phase de soumission à des désirs démiurgiques dans un premier temps pour tendre vers une collaboration plus équilibrée où les tensions deviennent des frictions servant l'accomplissement de l'écriture filmique. Ce phénomène, nous l'avons dit, se produit à l'occasion de Cría cuervos et surtout Elisa, vida mía. Dans les deux derniers films, los Ojos vendados et Mamá cumple cien años, les récits ne relèvent pas de la même complexité et, surtout dans le cas du premier, reviennent à des structures déjà à l'œuvre dans des films précédents comme Ana y los lobos, où la voix over narratrice de l'actrice n'apparaît quasiment plus, comme si cette dernière revenait à un statut plus classique de simple interprète. Ces films semblent alors ressentir le besoin de présenter à nouveau la voix et l'accent de Geraldine Chaplin comme étrangers, comme si une voix au cinéma n'était pas tant perçue comme étrangère en raison d'un accent mais surtout d'un éloignement de l'acteur de l'élan qui a mené la création de l'œuvre. 


\section{BIBLIOGRAPHIE}

Livres

BRASÓ Enrique, Carlos Saura, Madrid, Taller de ediciones Josefina Betancor, 1974.

CASTRO Antonio, El cine español en el banquillo, Valencia, Antonio Torres, 1974.

CHÂTEAUVERT Jean, Des mots à l'image : la voix over au cinéma, Québec, Nuit blanche éditeur, Paris, Méridiens Klincksieck, 1996.

CHION Michel, La voix au cinéma, Paris, Cahiers du cinéma, 1993.

FLEISCHER Alain, L'accent, une langue fantôme, Paris, Seuil, 2005.

GOLIOT-LÉTÉ Anne, Francis Vanoye, Précis d'analyse filmique, Paris, Armand Colin, 2009.

MORIN Edgar, Les stars, Paris, Seuil, 1972.

NACACHE Jacqueline, L'acteur de cinéma, Paris, Nathan, 2003.

NAREMORE James, Acting in cinema, Berkeley, L.A., London, University of California Press, 1988.

OMS Marcel, Carlos Saura, Paris, Edilig, 1981.

SÁNCHEZ VIDAL Agustín, El cine de Carlos Saura, Zaragoza, Caja de ahorros de la Inmaculada, 1988.

SHINGLER Martin, Star Studies, A Practical Guide, London, Palgrave Macmillan, BFI, 2012.

Thèse non publiée

BARTHOLOMEW Gail, « The Films of Carlos Saura 1959-1980 », Ph. D, Northwestern University, 1982.

Recueils

AMIEL Vincent, FARÇY Gérard-Denis, LUCET Sophie, SELLIER Geneviève (dir.), Dictionnaire critique de l'acteur, Rennes, Presses Universitaires de Rennes, 2012.

BLANCO Jesús, COLLADO Ignacio (dir.), De Saura, Córdoba, Filmoteca de Andalucía, Junta de Andalucía, 1993.

BONITZER Pascal, «Les silences de la voix », Le regard et la voix, Paris, Union générale d'éditions, 1976.

DOANE Mary Ann, « The Voice in the Cinema: The Articulation of Body and Space », dans WEIS Elizabeth, BELTON John (dir.), Film Sound: Theory and Practice, New York, Columbia University Press, 1985, pp. 33-50.

HERNÁNDEZ LES Juan A., « Carlos Saura y el cine (1959-1983) », dans Carmen Rodríguez Fuentes (dir.), Desmontando a Saura, Barcelona, Málaga, Luces de Gálibo, 2013, pp. 127-143.

LEMARIÉ Yannick, « Acteur, acté et forme », dans Jean-Louis Libois, Sophie Lucet (dir.), Double jeu, l'acteur créateur, ${ }^{\circ} 1$, Caen, Presses universitaires, 2003, pp. 25-34.

SOILA Tytti (dir.), Stellar Encounters: Stardom in Popular European Cinema, New Barnet and Hertfordshire, John Libbey, 2009. 


\section{NOTES}

1. Peppermint Frappé (1967), Stress es tres, tres (1968), la Madriguera (1969), Ana y los lobos (Anna et les loups, 1973), Cría cuervos... (1976), Elisa, vida mía (Elisa, mon amour, 1977), los Ojos vendados (les Yeux bandés, 1978) et Mamá cumple cien años (Maman a cent ans, 1979).

2. Ce n'est qu'à partir des années 1980 que Carlos Saura s'intéressera à la culture latinoaméricaine (Antonieta, 1982, El Dorado, 1988, Tango, 1998) et même portugaise (Fados, 2007).

3. Doctor Zhivago (Docteur Jivago, David Lean, 1965), Nashville (Robert Altman, 1975), Buffalo Bill and the Indians, or Sitting Bull's History Lesson (Buffalo Bill et les indiens, Robert Altman, 1976), Noroît (Jacques Rivette, 1976), Roseland (James Ivory, 1977), A Wedding (Un mariage, Robert Altman, 1978), le Voyage en douce (Michel Deville, 1980), la Vie est un roman (Alain Resnais, 1982), l'Amour par terre (Jacques Rivette, 1984), I Want to Go Home (Alain Resnais, 1989).

4. Rappelons l'explication de ce concept par James Naremore qui prend appui sur Stephen Heath : «[...] nous constatons généralement que la présence d'un acteur dans un film semble entrer en correspondance avec celle de son rôle. Pour des raisons similaires, on constate souvent des différences entre son image médiatique et le personnage, ou des changements dans le profil des rôles joués par une star. Ces perceptions peuvent participer à la structure et à la signification d'un film, même si elles sont soumises à des fluctuations dans le temps. Stephen Heath a remarqué que les films "jouent sur l'écart entre le rôle et la personne avec une importance variable". La grande ironie de cette situation est que "ce qui est en question est, en règle générale, la personne en tant que persona, c'est-à-dire la personne comme une image précisément cinématographique ; le corps humain se retrouvant réduit à cela"», James Naremore, Acting in cinema, Berkeley, L.A., London, University of California Press, 1988, p. 263. Pour la citation: Stephen Heath, Questions of Cinema, Bloomington, Indiana University Press, 1981, p. 181.

5. Agustín Sánchez Vidal, El cine de Carlos Saura, Zaragoza, Caja de ahorros de la Inmaculada, 1988, pp. 51-52.

6. Cité dans Gail Bartholomew, « The Films of Carlos Saura 1959-1980 », Ph. D, Northwestern University, 1982, p. 4.

7. Comme le reconnut Carlos Saura à Agustín Sánchez Vidal, cette première collaboration avec Geraldine Chaplin fut riche en tensions : « Geraldine se limita à son travail, sans la collaboration que nous aurions ensuite sur des films comme la Madriguera. Et, parfois, avec des discussions très violentes parce qu'elle n'était pas d'accord avec des choses qui étaient espagnoles et qu'elle ne comprenait pas bien », dans Agustín Sánchez Vidal, El cine de Carlos Saura, Zaragoza, Caja de ahorros de la Inmaculada, 1988, p. 54. Précisons que, dans les ouvrages concernant Carlos Saura, nous n'avons pas trouvé de témoignages de Geraldine Chaplin.

8. Bien entendu, cette décision n'est pas dénuée de réalisme, dans la mesure où la britannique Elena peut légitimement s'exprimer avec un accent, ce qui serait incohérent dans le cas de l'espagnole Ana.

9. Jacqueline Nacache, L'acteur de cinéma, Paris, Nathan, 2003, p. 61.

10. Yannick Lemarié, « Acteur, acté et forme », dans Jean-Louis Libois, Sophie Lucet (dir.), Double jeu, l'acteur créateur, $\mathrm{n}^{\circ} 1$, Caen, Presses universitaires, 2003, pp. 25-34, pp. 27-28. 
11. Edgar Morin, Les stars, Paris, Seuil, 1972, p. 110.

12. «Stress es tres tres est né de la collaboration avec Geraldine ; c'est-à-dire que, à partir de ce moment, il est rare que je fasse un film sans Geraldine », Carlos Saura dans Enrique Brasó, Carlos Saura, Madrid, Taller de ediciones Josefina Betancor, 1974, p. 198. « la Madriguera doit beaucoup à Geraldine, parce que beaucoup de choses qui sont dans le film sont des choses vécues par elle, ou qu'on lui a racontées [...] la Madriguera est une collaboration parfaite entre trois personnes [avec le coscénariste Rafael Azcona] », ibid., p. 236.

13. Martin Shingler, Star Studies, A Practical Guide, London, Palgrave Macmillan, BFI, 2012, p. 80. Pour la citation : Tytti Soila (dir.), Stellar Encounters : Stardom in Popular European Cinema, New Barnet and Hertfordshire, John Libbey, 2009, p. 17.

14. Vincent Amiel, Gérard-Denis Farçy, Sophie Lucet, Geneviève Sellier (dir.), Dictionnaire critique de l'acteur, Rennes, Presses Universitaires de Rennes, 2012, p. 241.

15. Marcel Oms observe que « dans chacun de ces films, à un moment donné, une danse anime le corps féminin, meut l'objet du désir et cristallise l'instant où tout va basculer [...] L'intrusion d'un corps étranger déclenche une série de phénomènes d'adaptation, de possession puis de rejet mortel ». Marcel Oms, Carlos Saura, Paris, Edilig, 1981, p. 44. 16. Ce plan rappelle l'analyse de Michel Chion qui reprend l'idée du cinéaste démiurge : «L'histoire est toujours la même : un homme [...] veut mettre sous son pouvoir, capturer dans la "cage de l'écran" comme dit Bazin, une voix féminine, et peut-être aussi la jouissance que cette voix lui signifie ", Michel Chion, La voix au cinéma, Paris, Cahiers du cinéma, 1993, p. 85.

17. Vincent Amiel, Gérard-Denis Farçy, Sophie Lucet, Geneviève Sellier (dir.), Dictionnaire critique de l'acteur, Rennes, Presses Universitaires de Rennes, 2012, p. 242.

18. Martin Shingler, Star Studies, A Practical Guide, London, Palgrave Macmillan, BFI, 2012, p. 160.

19. Michel Chion, La voix au cinéma, Paris, Cahiers du cinéma, 1993, p. 139.

20. Carlos Saura : «Au début, j'étais très réticent à tourner en son direct. Ce fut Geraldine, qui y est habituée, qui me convainquit que c'était préférable », Antonio Castro, El cine español en el banquillo, Valencia, Antonio Torres, 1974, p. 398.

21. Alain Fleischer, L'accent, une langue fantôme, Paris, Seuil, 2005, p. 30.

22. Cependant, dans la mesure où il s'agit de la reprise des personnages de Ana y los lobos, le phénomène n'a pas la même importance que dans los Ojos vendados.

23. « [...] un autre espace-temps que celui qui est représenté à l'écran », Anne Goliot-Lété, Francis Vanoye, Précis d'analyse filmique, Paris, Armand Colin, 2009, p. 39. Cet espacetemps renvoie, dans Cría cuervos..., au présent d'Ana adulte, vingt ans après les faits racontés. Dans Elisa, vida mía, cet espace-temps est moins identifiable et peut être contemporain à l'histoire et avoir lieu dans la maison du père ou être postérieurs.

24. Nous reprenons la terminologie de Jean Châteauvert et entendons la voix over comme une voix appartenant à un autre espace-temps que celui de l'écran, en opposition à une voix off qui serait une voix hors-champ. Cette terminologie s'oppose à celle, plus courante, qui identifie la voix over de Châteauvert comme off et donc nomme voix hors champ la voix off de ce dernier. Lire Anne Goliot-Lété, Francis Vanoye, op.cit., p. 39-40. 25. Cette lecture est appuyée par les propos de Carlos Saura : «Ce n'est pas toujours facile de travailler avec elle [Geraldine Chaplin] parce qu'elle a une forte personnalité et parfois elle se rebelle contre celui qui prétend la contrôler et la dominer, bien que ce soit dans ces moments de rébellion un peu infantile contre la tyrannie paternelle que j'exerce de temps en temps, qu'elle met en valeur toute sa sensibilité, dépassant ainsi mes attentes. », 
Jesús Blanco, Ignacio Collado (dir.), De Saura, Córdoba, Filmoteca de Andalucía, Junta de Andalucía, 1993, p. 59.

26. Marguerite Duras dans Pascal Bonitzer, «Les silences de la voix », Le regard et la voix, Paris, Union générale d'éditions, 1976, p. 44.

27. Rappelons que Saura lui-même évoque (note 25) son rôle dans cette collaboration, comme celle de l'autorité paternelle.

28. Jean Châteauvert, Des mots à l'image : la voix over au cinéma, Québec, Nuit blanche éditeur, Paris, Méridiens Klincksieck, 1996, p. 117. Jean Châteauvert cite : Pascal Bonitzer, «Les silences de la voix », Le regard et la voix, Paris, Union générale d'éditions, 1976;

Mary Ann Doane, «The Voice in the Cinema: The Articulation of Body and Space », dans Elizabeth Weis, John Belton (dir.), Film Sound: Theory and Practice, New York, Columbia University Press, 1985, pp. 33-50.

29. Juan $A$. Hernández Les associe également ce phénomène à la voix de l'actrice et parle d'un apport expressionniste : «Dans les films de Saura, ce qu'on a toujours désigné comme le réel imaginaire, ou le fantastique, naît souvent d'une confrontation purement expressionniste. L'expressionnisme saurien, dont on n'a jamais parlé, est produit par l'introduction d'un agent externe : la diction singulière, différente, monstrueuse, de Geraldine Chaplin », Juan A. Hernández Les, « Carlos Saura y el cine (1959-1983) », dans Carmen Rodríguez Fuentes (dir.), Desmontando a Saura, Barcelona, Málaga, Luces de Gálibo, 2013, pp. 127-143, p. 131.

\section{RÉSUMÉS}

Dans la filmographie de Carlos Saura, Geraldine Chaplin a permis l'apparition du féminin en en incarnant une représentation dichotomique qui repose grandement sur la voix dans la mesure où, selon les œuvres, l'actrice est doublée ou non. Ce doublage artificiel en raison de la présence intermittente de la vraie voix de Chaplin, aurait pu empêcher la reconnaissance de son talent, en démontrant l'inutilité de l'actrice face à un cinéaste qui maîtrise la création du personnage au montage son. Cependant, Chaplin s'affirme dans l'écriture cinématographique des œuvres dès La madriguera - film qu'elle coécrit et où elle conserve sa voix - en conférant à la dualité de son personnage une nouvelle maturité. La voix de Chaplin, nécessaire à l'affirmation de sa persona, révèle son corps, ses capacités actorales et appelle une nouvelle écriture filmique. En y assumant toutes les fonctions possibles de la voix - in, over, lectrice, narratrice... -, elle devient pleinement cinématographique et produit des frictions énonciatrices qui, tout en relevant d'une collaboration non dénuée d'oppositions entre cinéaste et actrice, accompagnent l'écriture filmique des œuvres vers son accomplissement.

Geraldine Chaplin opened the way for the apparition of the feminine in Carlos Saura's filmography, in a dichotomous rôle which relies to a great extent upon the voice in that, depending on the different films, the actress's voice is dubbed or not. The dubbed voice, which is artificial due to the presence of Chaplin's real voice in certain films, could have proved a stumbling block to the recognition of her talent by demonstrating the helplessness of an actress faced with a film-maker who controls the creation of a character via the sound editing process. However, Chaplin asserts herself in the cinematographic text of the works from La Madriguera - a 
film she co-wrote and in which she retains her own voice - onwards, bestowing a new maturity upon the duality of her character. Chaplin's voice, which is necessary to the assertion of her persona, reveals her body, her capabilities as an actress and ushers in a new form of film-writing. By assuming all the different functions of the voice - in, over, as a reader and as a narrator...- she becomes fully cinematographic and produces enunciative frictions which, whilst revealing a collaboration (far from free of contrasts) between actress and film-maker, escort the film-writing of these works towards their accomplishment.

\section{INDEX}

Mots-clés : son direct, doublage, postsynchronisation, accent, persona, narration, C. Saura (1932-) et G. Chaplin (1944-)

Schlüsselwörter : son direct, doublage, postsynchronisation, accent, persona, narration, C. Saura (1932-) et G. Chaplin (1944-)

Palabras claves : son direct, doublage, postsynchronisation, accent, persona, narration, C. Saura (1932-) et G. Chaplin (1944-)

Keywords : son direct, doublage, postsynchronisation, accent, persona, narration, C. Saura (1932-) et G. Chaplin (1944-)

\section{AUTEUR}

\section{ARNAUD DUPRAT DE MONTERO}

Ancien élève du CLCF (Conservatoire Libre du Cinéma Français), Arnaud Duprat de Montero est maître de conférences à l'université Rennes 2 . Ses recherches portent sur les derniers films de Luis Buñuel avec une approche transculturelle, ainsi que sur la représentation du personnage à l'écran et la part auctoriale de l'acteur dans la création cinématographique. Il est l'auteur d'articles sur Luis Buñuel, Jean-Claude Carrière, Carlos Saura, Raúl Ruiz, Pedro Almodóvar, Víctor Erice, Jaime Rosales, Isabelle Adjani et Ava Gardner. Il a publié en 2011 Le dernier Buñuel, aux Presses universitaires de Rennes (collection « Le spectaculaire »), a participé en 2012 à l'édition blu-ray de Cet obscur objet du désir (L. Buñuel, 1977) chez Studiocanal et a présenté en 2013 Isabelle Adjani, un mythe de l'incarnation aux éditions Le bord de l'eau (collection « Cinémythologies »). 